\title{
Multi-objective Quantum Moth Flame Optimization for Clustering
}

\author{
Yassmine Soussi $^{[1-2]}$ (D), Nizar Rokbani ${ }^{[1-3]}$, Ali Wali ${ }^{[1]}$, Adel.M Alimi ${ }^{[1]}$ \\ ${ }^{1}$ REGIM-Lab: REsearch Groups in Intelligent Machines, University of Sfax, National \\ Engineering School of Sfax (ENIS), BP 1173, Sfax, 3038, Tunisia \\ ${ }^{2}$ University of Sousse, ISITCom, 4011, Hammem Sousse, Tunisia \\ ${ }^{3}$ University of Sousse, High Institute of Applied Science and technology of Sousse, Tunisia \\ \{yassmine.soussi, nizar.rokbani, ali.wali, adel.alimi\}@ieee.org \\ Accepted Chapter book : Enabling Machine Learning Applications \\ in Data Science, Algorithms for Intelligent Systems, Springer.
}

\begin{abstract}
This paper defines a new Moth-Flame optimization version with Quantum behaved moths, QMFO. The multi-objective version of QMFO (MOQMFO) is then applied to solve clustering problems. MOQMFO used three cluster validity criteria as objective functions (the I-index, Con-index and Sym-index) to establish the multi-objective clustering optimization. This paper details the proposal and the preliminary obtained results for clustering real-life datasets (including Iris, Cancer, Newthyroid, Wine, LiverDisorder and Glass) and artificial datasets (including Sph_5_2, Sph_4_3, Sph_6_2, Sph_10_2, Sph_9_2, Pat 1, Pat 2, Long 1, Sizes 5, Spiral, Square 1, Square 4, Twenty and Fourty). Compared with key multi-objectives clustering techniques, the proposal showed interesting results essentially for Iris, Newthyroid, Wine, LiverDisorder, Sph_4_3, Sph_6_2, Long 1, Sizes 5, Twenty and Fourty; and was able to provide the exact number of clusters for all datasets.
\end{abstract}

Keywords: Moth Flame Optimization Algorithm (MFO), Quantum hybridization, Quantum Behaved MFO (Q-MFO), Multi-Objective Quantum Moth Flame Optimization (MOQMFO), Multi-Objective Clustering Optimization (MOCO).

\section{Introduction}

Clustering consist in dividing a dataset into $\mathrm{k}$ clusters and this based on the similarities of objects belonging to each cluster (Jain, 2010); The clustering problem can be defined as a mono-objective optimization problem with one objective function presented by one cluster validity criteria.

Taking into account that the classification problems have become more complex and different cluster validity criteria have appeared, it has therefore become necessary 
to consider the clustering as a multi-objective optimization problem (Mukhopadhyay et al., 2015) (Cunha et al., 2017) with different objective functions presented by different cluster validity criteria.

Various multi-objective clustering techniques in the literature have been proposed to solve the multi-objective clustering optimization problem including: The MultiObjective Clustering with automatic determination of $\mathrm{K}$ ( $\mathrm{K}$ is the number of clusters), MOCK, proposed by (Matake et al., 2007); The Variable String Length Point Symmetry-Based, VGAPS, proposed by (Bandyopadhyay \& Saha, 2008), The two multi-objective simulated annealing methods, GenClustMOO and GenClustPESA2, proposed by (Saha \& Bandyopadhyay, 2013); The multi-objective clustering algorithm based on artificial bee optimization, cOptBees-MO, proposed by (Cunha et al., 2017). The multi-objective Particle Swarm Optimization based on Simulated Annealing algorithm, MOPSOSA, proposed by (Abubake et al., 2015) . These multiobjective clustering techniques are used as comparatives algorithms with the proposal.

In this study, MOQMFO algorithm used 3 cluster validity indices as objective functions: the I-Index (Cunha et al., 2017) (Saha \& Bandyopadhyay, 2013), the SymIndex (Cunha et al., 2017) (Bandyopadhyay \& Saha, 2007), and the Con-Index (Cunha et al., 2017) (Saha \& Bandyopadhyay, 2012); the optimization of these objectives functions aims to detect the correct number of clusters and find a good clustering solution for each dataset. Here, F-measure (Saha \& Bandyopadhyay, 2013) (Fung et al., 2003) is the external validation measure used to evaluate and compare the final clustering solutions obtained by MOQMFO and its competitors.

This paper is organized as follow: In section 2 MFO algorithm and QMFO algorithm were presented. In section 3 the Multi-objective form of QMFO (MOQMFO) was introduced. In section 4 an application of MOQMFO in the multiobjective clustering optimization domain was made. Section 5 presents MOQMFO flowchart. In section 6, some experiments have been carried out. And finally, in section 7 , a conclusion with some perspectives was performed.

\section{The Quantum Behaved MFO (Q-MFO)}

\subsection{Moth Flame Optimization (MFO)}

MFO is a new metaheuristic population-based method, it was proposed by (Mirjalili, 2015) based on the flying mode of moths while looking for light sources (Flames). In a given problem, the positions of Moths and Flames are considered solutions. Moths present the actual solutions (actual positions) while Flames presents the best solutions (best positions). The numbers of moths "nMoths" and the number of flames "nFlames" are equal (nMoths = nFlames). When moving to a Flame, Moth changes its position using a logarithmic-spiral-function; the position of a moth is then updated using Eq.1.

$$
S\left(\text { Moth }_{i}, \text { Flame }_{j}\right)=D_{i} \cdot e^{b t} \cdot \operatorname{Cos}(2 \pi t)+\text { Flame }_{j}
$$


- S defines the Spiral function used by the Moth while moving;

- $D_{\mathrm{i}}$ is the Euclidian distance between the $\mathrm{i}$-th moth and the $\mathrm{j}$-th flame, illustrated in Eq.2.

$$
D_{i}=\left|F_{j}-M_{i}\right|
$$

- $\quad \mathrm{b}$ is a constant for establishing the logarithmic-spiral form;

- $\mathrm{t}$ is a number chosen randomly from $[-1,1]$.

MFO parameters are simple and do not require a large adjustment that makes MFO algorithm simple, easy to implement and robust.

In the rest of this document we combined the quantum theory (Jun et al., 2006) (Yang \& Wang, 2004) (Dereli \& Köker, 2020) (Yu et al., 2020) into MFO and proposed a Quantum-behaved MFO (QMFO) algorithm. Next section will describe the application of the quantum technique in MFO.

\subsection{Quantum-behaved MFO (QMFO)}

The quantum mechanics is a new area that has been influenced by several factors namely: The atomic theory (Bohr, 1928) proposed by Bohr in 1928; the quantum mechanics (Heisenberg, 1929) discovered by Heisenberg; the wave mechanics (Wessels, 1979) discovered by Schrödinger. According to Feynman (Feynman, 1982), the simulation of quantum mechanical systems is continuous and fast with quantum computers than with ordinary computers. Following the evolution of quantum computing applications over the past three decades, the concept of quantum computing, once thought to be a mere theoretical alternative, has become a real alternative (Jiacun, 2012).

The methods of evolutionary computation and swarm intelligence are classifications of population-based methods known as optimization algorithms. These methods have become increasingly in demand in research communities over the past 20 years. In 1995, particle swarm optimization (PSO) technique was introduced by (Kennedy \& Eberhart, 1995) as an optimization algorithm, it was inspired by the social behavior of particles (a swarm is a group of particles).

Taking into consideration the creation of quantum-based optimization techniques inspired by the combination of three area: the quantum computing, the mathematics and the computer science; several hybridization of quantum theory and optimization algorithms have been developed namely the quantum particle swarm optimization (QPSO) how is a variant of Particle Swarm Optimization proposed in (Sun et al., 2004) (Sun et al., 2004) (Sun et al., 2005). By referring to these previous studies it has been proven that QPSO shows good convergence and good performance compared to PSO. In (Jun et al., 2006) authors explored the applicability of QPSO in the data clustering domain and proved that QPSO performance is better than PSO performances and that is because the global convergence behavior of QPSO.

In this paper, Quantum-Behaved MFO (QMFO) is a search technique inspired by QPSO. The new quantum equations introduced in QMFO are gather to those used in QPSO clustering algorithm (Jun et al., 2006). As QPSO, QMFO guarantees global 
convergence and makes Moths more dynamic with wave function instead of their positions and the positions of theirs corresponding Flames. A new variable is introduced called mean best position "mbest"; this variable has a role in improving Q-MFO functionality.

Since the flames present the best "pbest" positions of the moths; The best pbest of these flames, called the best overall "gbest" and the best average flame among these flames, called "mbest" are calculated from the set of flames using Eq. 3.

$$
\text { mbest }=\frac{1}{\text { nFlames }} \sum_{j=1}^{n \text { Flames }}(\text { Flame }(j))
$$

In QMFO, the moth moves and changes its position using Eq. 5.

$$
\begin{gathered}
\text { Flame }_{j}=\phi \cdot \text { Flame }_{j}+(1-\phi) \cdot \text { gbest } \\
\text { Moth }_{\mathrm{i}}=\mathrm{D}_{\mathrm{i}} \cdot \mathrm{e}^{\mathrm{bt}} \cdot \operatorname{Cos}(2 \pi \mathrm{t})+\text { Flame }_{\mathrm{j}} \pm \alpha \cdot \mid \text { mbest }- \text { Moth }_{\mathrm{i}} \mid \cdot \ln \left(\frac{1}{\mathrm{u}}\right)
\end{gathered}
$$

The convergence behavior of the Moths in QMFO is influenced by:

- The new Flame $j_{j}$ calculated in Eq. 4, who is a randomly determined point between Flame ${ }_{j}$ and gbest, it is the attractor of Moth ${ }_{i}$;

- $\phi$ and $\mathrm{u}$ which are two random numbers distributed uniformly in the interval $[0,1]$;

- $\quad \alpha$, named "Contraction-Expansion-Coefficient", who is a relative parameter to QMFO algorithm.

\section{Multi-objective QMFO (MOQMFO)}

MOQMFO is the multi-objective form of QMFO algorithm, it combines the characteristics of the QMFO and the Multi-Objective Optimization (Deb, 2001) (Freitas, 2004).

MOQMFO uses the concept of "non-dominance-Pareto" to store the best solutions obtained during each generation into a "Repository": the Repository is a set of non dominated solutions called "pareto set solutions".

\section{$4 \quad$ MOQMFO for clustering}

In the Multi-Objective Clustering Optimization (Mukhopadhyay et al., 2015) area, a moth or flame is presented by a matrix $(\mathrm{k} * \mathrm{~d}) ; \mathrm{k}$ and $\mathrm{d}$ present respectively the number of clusters and the number of features related to the dataset: As showed in Eq. 6, each line-vector in this matrix presents a cluster center. 


$$
\operatorname{Moth}_{\mathrm{i}}=\left[\begin{array}{c}
\mathrm{C}_{\mathrm{i} 1} \\
\cdot \\
\cdot \\
\cdot \\
\mathrm{C}_{\mathrm{ij}} \\
\cdot \\
\cdot \\
\cdot \\
\mathrm{C}_{\mathrm{ik}}
\end{array}\right], \quad \mathrm{i}=[1, \ldots, \text { nMoths }]
$$

To calculate the fitness of each moth or flame, a combination of three objective functions is used. In our case clusters validity index (the I-Index (Cunha et al., 2017) (Saha \& Bandyopadhyay, 2013), the Sym-Index (Cunha et al., 2017) (Bandyopadhyay \& Saha, 2007), and the Con-Index (Cunha et al., 2017) (Saha \& Bandyopadhyay, 2012)) are used as objectives functions.

The architecture of our proposed Methodology MOQMFO for clustering is presented in Fig.1.

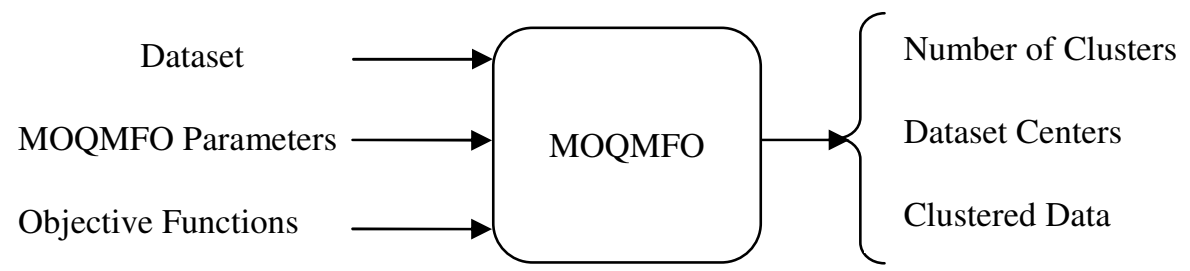

Fig.1. MOQMFO Architecture.

\section{MOQMFO flowchart}

After selecting the range of values of $\mathrm{k}$ (number of clusters) related to the dataset using MFO based Mean-distance clustering algorithm, the MOQMFO algorithm was run $n$ ( $n=$ number of runs) times for each value of $k$, and in each run, the optimal " $F$ Mesaure" as well as its corresponding number of clusters and optimal position of clusters centers "Best-Position" are selected and then stored. In the end of all runs, a set of $\mathrm{n}$ values of F-Measure with its corresponding number of clusters and positions of clusters centers is obtained; the higher value of F-Measure, with its corresponding "Best-Position" of clusters centers and its corresponding value of $\mathrm{k}$, is then selected from this set.

The flowchart of the proposed method, MOQMFO for clustering is detailed in Fig.2. MOQMFO flowchart is based on four phases: The initialization, the optimization, the decision making1 and the decision making2.

The second and third phases are repeated $\mathrm{n}$ times ( $\mathrm{n}$ equals to MaxIteration). 
As input we have the dataset, the number of clusters (k), the number of Moths (nMoth), number of Flames (nFlame), the maximum number of iteration (MaxIteration) and the repository size (nRep).

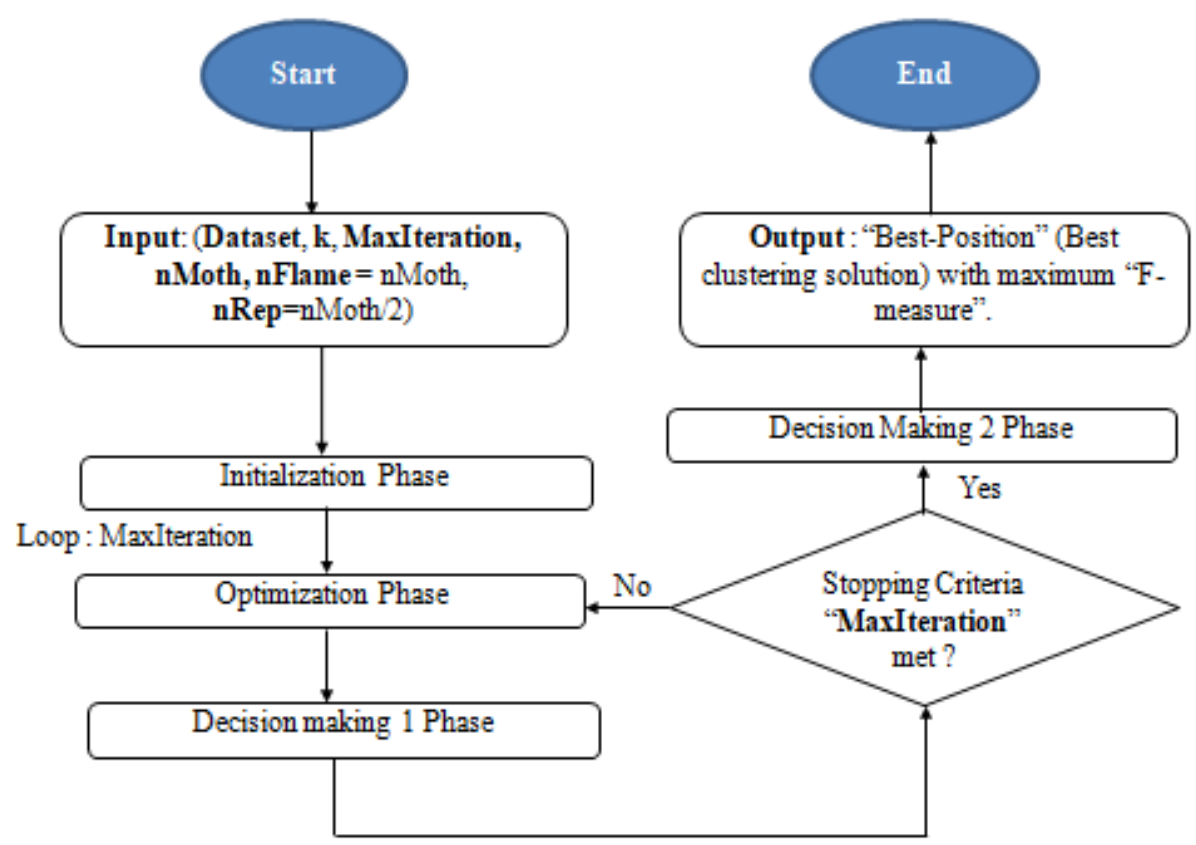

Fig.2. MOQMFO Flowchart.

The flowchart of the Initialization phase, showed in Fig.3, consists in generating the initials moths, flames, and repository members.

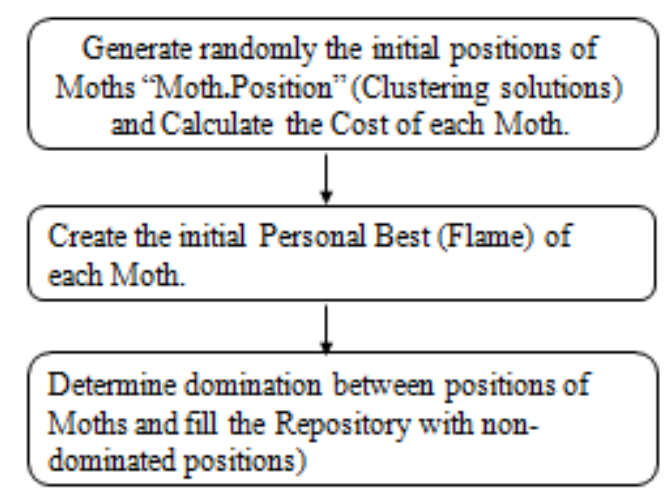

Fig.3. MOQMFO Initialization phase Flowchart. 
The "Optimization" consists in updating the Moths and flames positions and fill the repository.

The "Decision Making1" phase (red blocs): consist in selecting the best position with the best cost, in the pareto-set-solutions produced in each iteration, then calculating the F-measure obtained after the classification of the dataset using this best position which represents the clusters centers positions;

Fig.4 illustrates the flowchart of the "Optimization" phase and "Decision Making1" phase of the MOQMFO algorithm

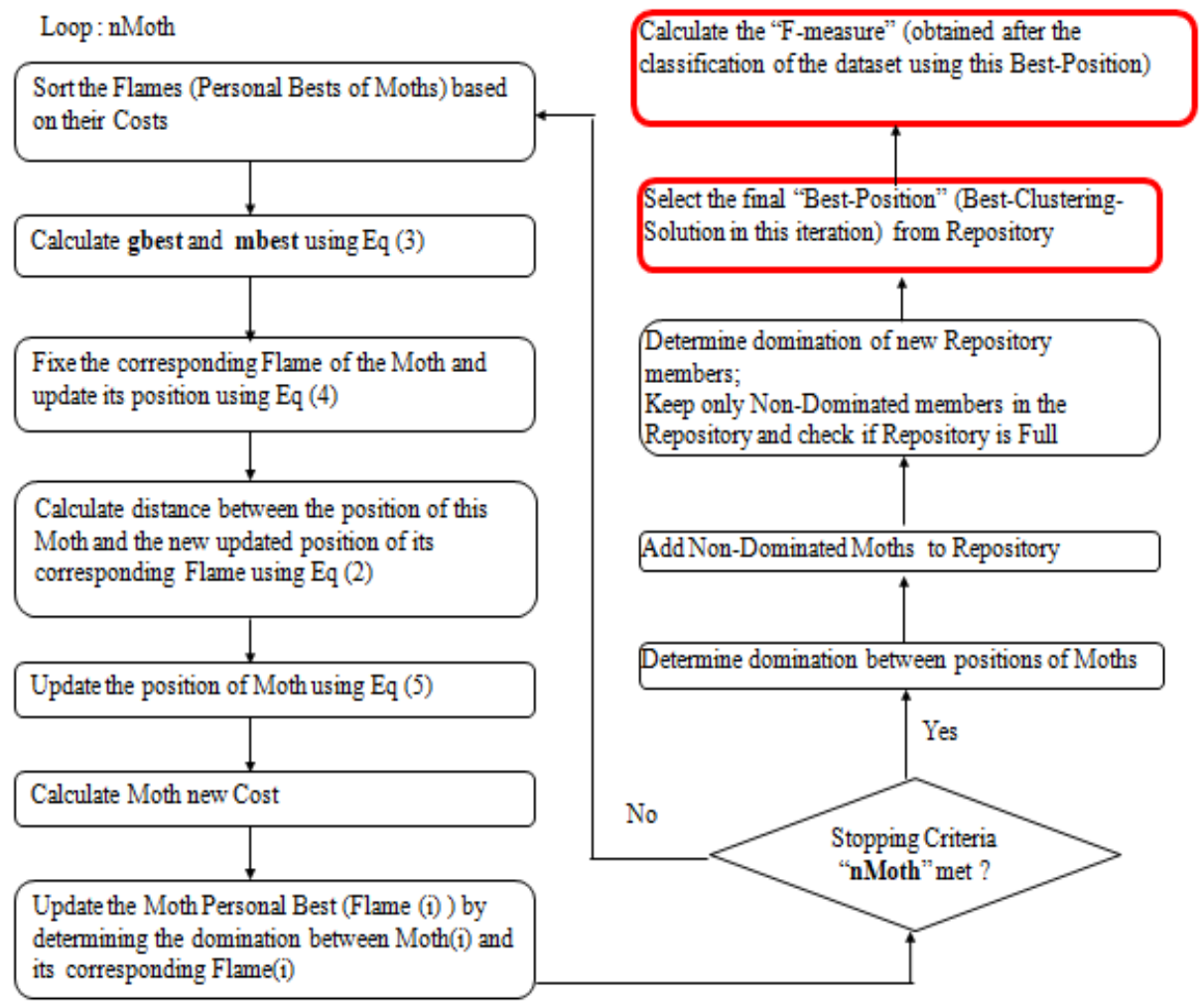

Fig.4. The flowchart of the "Optimization" phase and "Decision Making1" phase of the MOQMFO algorithm.

The "Decision making2" phase: consist in selecting the best position with the best "F-measure" in the set of "F-measure" produced in the end of all iterations. 


\section{Experiments}

\subsection{Datasets}

In the experimentations part, 20 datasets ,including Iris (Lichman, 2013), Cancer (Lichman, 2013), Newthyroid (Lichman, 2013), Wine (Lichman, 2013), LiverDisorder (Lichman, 2013), Glass (Lichman, 2013), Sph_5_2 (Bandyopadhyay \& Maulik, 2002), Sph_4_3 (Bandyopadhyay \& Maulik, 2002), Sph_6_2 (Bandyopadhyay \& Maulik, 2002) ,Sph_10_2 (Bandyopadhyay \& Pal, 2007), Sph_9_2 (Bandyopadhyay \& Pal, 2007), Pat 1 (Pal \& Mitra, 1994), Pat 2 (Pal \& Mitra, 1994), Long 1 (Handl \& Knowles, 2007), Sizes 5 (Handl \& Knowles, 2007), Spiral (Handl \& Knowles, 2007), Square 1 (Handl \& Knowles, 2007), Square 4, Twenty (Handl \& Knowles, 2007) and Fourty (Handl \& Knowles, 2007), are used to evaluate the performances of MOQMFO and the competing algorithms. These datasets are divided into two types: Real-life and artificial datasets. In Table 1, there is a representation of these datasets: number of cases (\#points), number of features (Dimension) and number of clusters, corresponding to each one.

Table 1. Description of datasets.

\begin{tabular}{|c|c|c|c|c|}
\hline \multicolumn{2}{|r|}{ Dataset } & \# Points & Dimension & \# Clusters \\
\hline \multirow{6}{*}{ 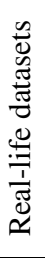 } & Iris & 150 & 4 & 3 \\
\hline & Cancer & 683 & 9 & 2 \\
\hline & Newthyroid & 215 & 5 & 3 \\
\hline & LiverDisorder & 345 & 6 & 2 \\
\hline & Glass & 214 & 9 & 6 \\
\hline & Wine & 178 & 13 & 3 \\
\hline \multirow{14}{*}{ 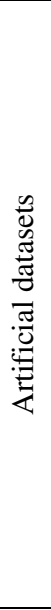 } & Sph_5_2 & 250 & 2 & 5 \\
\hline & Sph_4_3 & 400 & 3 & 4 \\
\hline & Sph_6_2 & 300 & 2 & 6 \\
\hline & Sph_10_2 & 500 & 2 & 10 \\
\hline & Sph_9_2 & 900 & 2 & 9 \\
\hline & Pat 1 & 557 & 2 & 3 \\
\hline & Pat 2 & 417 & 2 & 2 \\
\hline & Long 1 & 1000 & 2 & 2 \\
\hline & Sizes 5 & 1000 & 2 & 4 \\
\hline & Spiral & 1000 & 2 & 2 \\
\hline & Square 1 & 1000 & 2 & 4 \\
\hline & Square 4 & 1000 & 2 & 4 \\
\hline & Twenty & 1000 & 2 & 20 \\
\hline & Fourty & 1000 & 2 & 40 \\
\hline
\end{tabular}




\subsection{Scenario}

MOQMFO was executed 30 times with the input parameters shown in Table 2:

Table 2. MOQMFO Parameters

\begin{tabular}{ll}
\hline Parameter & Value \\
\hline Number-moths & 50 \\
Number-flames & 50 \\
Repository-size & 25 \\
Number-iterations & 100 \\
\hline
\end{tabular}

F-measure (Saha \& Bandyopadhyay, 2013), (Fung et al., 2003) is the external validation measure used to evaluate and compare the final clustering solutions obtained by MOQMFO and the algorithms used for comparison (GenClustMOO, MOCK, VGAPS, GenClustPESA2, cOptBees-MO and MOPSOSA).

Higher values of F-Measure imply better clustering, and when F-Measure reaches the value 1 the clustering is optimal.

\subsection{Results and discussion}

The MOQMFO algorithm was applied with 6 real-life datasets and 14 artificial datasets. Comparisons are based on F-measure values and number of clusters (k) obtained with MOQMFO and its competitors.

Obtained results, illustrated in Table 3, show that MOQMFO had a competitive performance when compared with the other multi-objective clustering techniques.

According to Table 3 MOQMFO algorithm provided the real number of clusters for all real-life and artificial datasets.

Compared with its competitors, MOQMFO gave the best values of F-Measure with the following datasets: Iris (F-Measure=1), Newthyroid (F-Measure=0.96), LiverDisorder (F-Measure=0.75), Wine (F-Measure=0.91), Sph_4_3 (F-Measure=1), Sph_6_2 (F-Measure=1), Long 1 (F-Measure=1), Sizes 5 (F-Measure=0.99), Twenty (F-Measure=1) and Fourty (F-Measure=1).

However MOQMFO provided acceptable values of F-Measure for the following cases: Cancer (F-Measure=0.91), Glass (F-Measure=0.60), Sph_5_2 (FMeasure=0.96), Sph_10_2 (F-Measure=0.96), Sph_9_2 (F-Measure=0.80), Pat 1 (FMeasure $=0.77)$, Pat $2(\mathrm{~F}-\mathrm{Measure}=0.82)$, Spirale $(\mathrm{F}-\mathrm{Measure}=0.65)$, Square 1 (FMeasure $=0.97)$ and Square $4($ F-Measure $=0.92)$.

The clustering results of the artificial datasets based on MOQMFO Clustering algorithm are presented in Fig.5: Sph_5_2 presented in Fig.5a, Sph_4_3 illustrated in Fig.5b, Sph_6_2 showed in Fig.5c, Sph_10_2 presented in Fig.5d, Sph_9_2 illustrated in Fig.5e, Pat 1 depicted in Fig.5f, Pat 2 illustrated in Fig.5g, Long 1 showed in Fig.5h, Sizes 5 presented in Fig.5i, Spiral showed in Fig.5j, Square 1 illustrated in Fig.5k, Square 4 depicted in Fig.5l, Twenty presented in Fig.5m and Fourty illustrated in Fig.5n. 
Table 3. F-measure values and number of clusters obtained with MOQMFO and the competing algorithms.

\begin{tabular}{|c|c|c|c|c|c|c|c|c|c|c|c|c|c|c|c|}
\hline \multirow[b]{2}{*}{ Dataset } & \multirow[b]{2}{*}{ \#Clusters } & \multicolumn{2}{|c|}{ MOPSOSA } & \multicolumn{2}{|c|}{ GenClustMOO } & \multicolumn{2}{|c|}{ GenClustPESA2 } & \multicolumn{2}{|c|}{ MOCK } & \multicolumn{2}{|c|}{ VGAPS } & \multicolumn{2}{|c|}{ cOptBee-MO } & \multicolumn{2}{|c|}{ MOQMFO } \\
\hline & & $\mathrm{k}$ & F- Measure & $\mathrm{k}$ & F-Measure & $\mathrm{k}$ & F- Measure & $\mathrm{k}$ & F- Measure & $\mathrm{k}$ & F- Measure & $\mathrm{k}$ & F- Measure & $\mathrm{k}$ & F-Measure \\
\hline Iris & 3 & 3 & 0.92 & 3 & 0.79 & 3 & 0.93 & 2 & 0.78 & 3 & 0.76 & 3 & 1.00 & 3 & 1.00 \\
\hline Cancer & 2 & 2 & 0.98 & 2 & 0.97 & 2 & 0.98 & 2 & 0.82 & 2 & 0.95 & 3 & 0.94 & 2 & 0.91 \\
\hline Newthyroid & 3 & 3 & 0.89 & 3 & 0.86 & 9 & 0.69 & 2 & 0.74 & 5 & 0.66 & 4 & 0.86 & 3 & 0.96 \\
\hline LiverDisorder & 2 & 2 & 0.69 & 2 & 0.67 & 5 & 0.60 & 2 & 0.67 & 2 & 0.70 & 2 & 0.67 & 2 & 0.75 \\
\hline Glass & 6 & 6 & 0.57 & 6 & 0.49 & 5 & 0.53 & 5 & 0.53 & 5 & 0.53 & 2 & 0.88 & 6 & 0.60 \\
\hline Wine & 3 & - & - & 3 & 0.71 & 13 & 0.44 & 3 & 0.73 & 6 & 0.62 & 2 & 0.65 & 3 & 0.91 \\
\hline$\overline{S p h \_5 \_2}$ & 5 & 5 & 0.98 & 5 & 0.97 & 5 & 0.94 & 6 & 0.91 & 5 & 0.55 & - & - & 5 & 0.96 \\
\hline $\mathrm{Sph} \_4 \_3$ & 4 & 4 & 1.00 & 4 & 1.00 & 4 & 1.00 & 4 & 1.00 & 4 & 1.00 & - & - & 4 & 1.00 \\
\hline Sph_6_2 & 6 & 6 & 1.00 & 6 & 1.00 & 6 & 1.00 & 6 & 1.00 & 6 & 1.00 & - & - & 6 & 1.00 \\
\hline Sph_10_2 & 10 & 10 & 0.99 & 10 & 0.99 & 12 & 0.94 & 6 & 0.72 & 7 & 0.76 & - & - & 10 & 0.96 \\
\hline Sph_9_2 & 9 & 9 & 0.92 & 9 & 0.69 & 8 & 0.66 & 9 & 0.73 & 9 & 0.49 & - & - & 9 & 0.80 \\
\hline Pat 1 & 3 & 3 & 1.00 & 3 & 0.95 & 3 & 0.95 & 10 & 0.55 & 4 & 0.42 & - & - & 3 & 0.77 \\
\hline Pat 2 & 2 & 2 & 1.00 & 2 & 1.00 & 2 & 1.00 & 11 & 0.55 & 4 & 0.59 & - & - & 2 & 0.82 \\
\hline Long 1 & 2 & 2 & 1.00 & 2 & 1.00 & 2 & 1.00 & 2 & 1.00 & 3 & 0.50 & - & - & 2 & 1.00 \\
\hline Sizes 5 & 4 & 4 & 0.98 & 4 & 0.97 & 3 & 0.88 & 2 & 0.80 & 5 & 0.82 & - & - & 4 & 0.99 \\
\hline Spiral & 2 & 2 & 1.00 & 2 & 1.00 & 2 & 1.00 & 3 & 0.95 & 6 & 0.38 & - & - & 2 & 0.65 \\
\hline Square 1 & 4 & 4 & 0.99 & 4 & 0.99 & 4 & 0.99 & 4 & 0.99 & 4 & 0.99 & - & - & 4 & 0.97 \\
\hline Square 4 & 4 & 4 & 0.94 & 4 & 0.92 & 4 & 0.88 & 4 & 0.90 & 2 & 0.93 & - & - & 4 & 0.92 \\
\hline Twenty & 20 & 20 & 1.00 & 20 & 1.00 & 24 & 0.95 & 20 & 1.00 & 20 & 0.48 & - & - & 20 & 1.00 \\
\hline Fourty & 40 & 40 & 1.00 & 40 & 1.00 & 40 & 0.98 & 40 & 1.00 & 2 & 0.10 & - & - & 40 & 1.00 \\
\hline
\end{tabular}




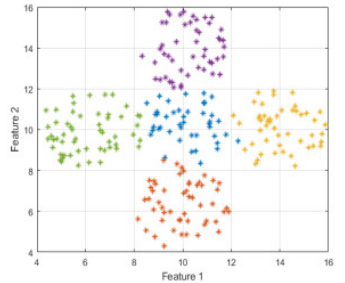

(a)

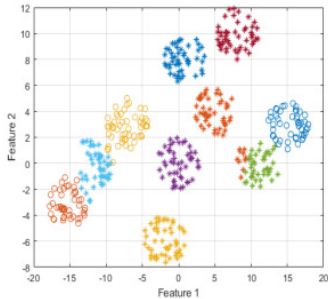

(d)

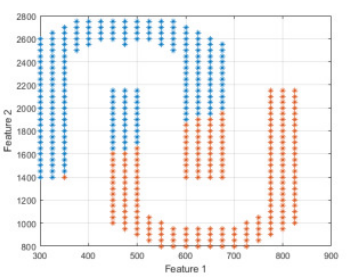

(g)

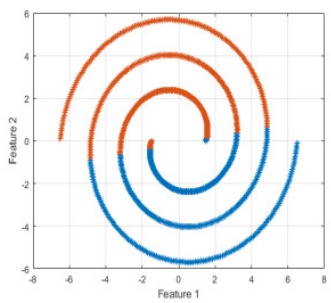

(j)

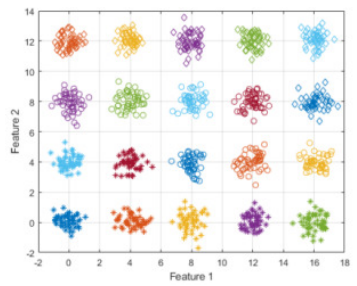

(m)

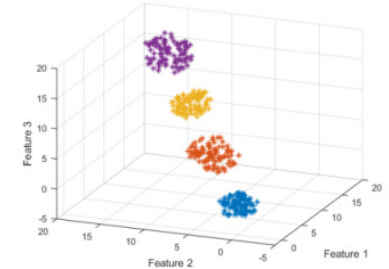

(b)

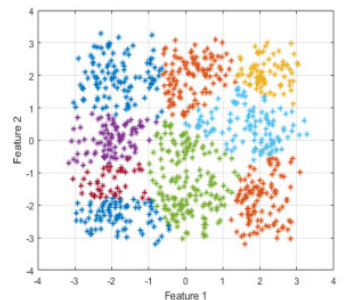

(e)

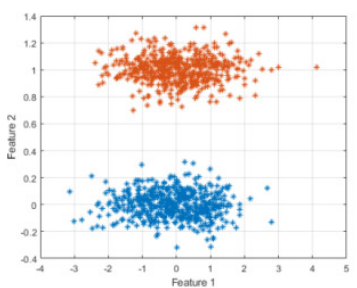

(h)

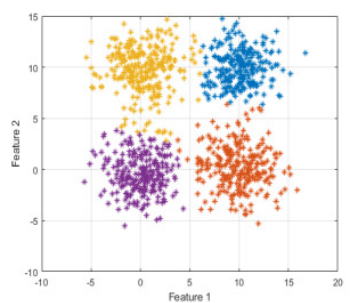

(k)

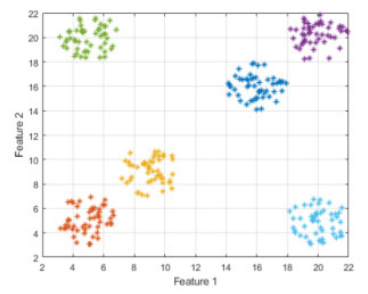

(c)

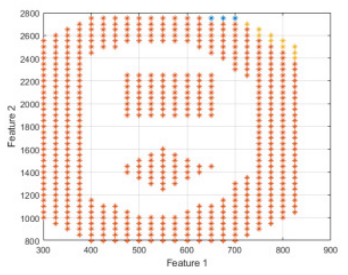

(f)

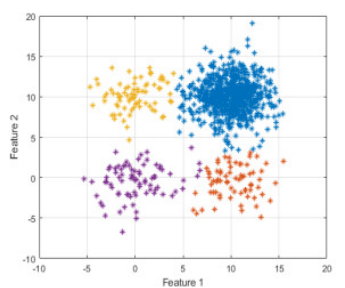

(i)

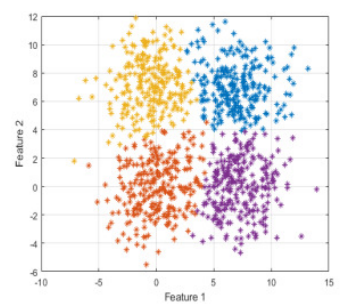

(l)

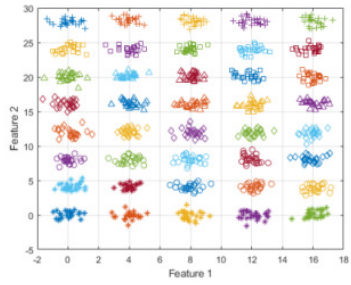

(n)

Fig.5.Graphical representations of artificial datasets based on MOQMFO clustering technique. 


\section{$7 \quad$ Conclusion and perspectives}

In this paper, a hybridization of quantum theory and the Moths Flames (QMFO) optimization algorithm is proposed, the multi-objective form of QMFO (MOQMFO) is then applied in multi-objective clustering optimization area.

To evaluate the performance of MOQMFO, a scenario based on three cluster validity criteria (I-index, Con-index and Sym-index), used as objective functions, is carried out. 20 datasets (including Iris, Cancer, Newthyroid, Wine, LiverDisorder, Glass, Sph_5_2, Sph_4_3, Sph_6_2, Sph_10_2, Sph_9_2, Pat 1, Pat 2, Long 1, Sizes 5, Spiral, Square 1, Square 4, Twenty and Fourty) and 6 multi-objective algorithms (GenClustMOO, MOCK, VGAPS, GenClustPESA2, cOptBees-MO and MOPSOSA) are used in this scenario. F-measure is the metric used in the evaluation of MOQMFO performances.

The results provided in the experiments part, show that MOQMFO had a high capacity to provide an optimal clustering solution, with the correct number of clusters over all datasets, and a competitive F-measure value comparing with other multiobjective clustering optimization algorithms.

Best results of MOQMFO are provided in Iris, Newthyroid, Wine, LiverDisorder, Sph_4_3, Sph_6_2, Long 1, Sizes 5, Twenty and Fourty datasets.

Our perspectives in this subject consist in improving MOQMFO performances on Glass, Sph-9-2, Pat 1, Pat 2 and Spiral datasets ; Using other cluster validity criteria as objective functions and introducing other new techniques for the MFO algorithm and apply them in the multi-objective clustering optimization area 


\section{$8 \quad$ References}

1. Abubake, A., Baharum, A. \& Alrefaei, M., 2015. Automatic clustering using multi-objective particle swarm and simulated annealing. PloS one, 10(7).

2. Bandyopadhyay, S. \& Maulik, U., 2002. Genetic clustering for automatic evolution of clusters and application to image classification. Pattern recognition, 35(6), pp.1197-208.

3. Bandyopadhyay, S. \& Pal, S.K., 2007. Classification and learning using genetic algorithms: applications in bioinformatics and web intelligence. Springer Science \& Business Media. .

4. Bandyopadhyay, S. \& Saha, S., 2007. GAPS: A clustering method using a new point symmetry-based distance measure. Pattern recognition, 40(12), pp.343051.

5. Bandyopadhyay, S. \& Saha, S., 2008. A point symmetry-based clustering technique for automatic evolution of clusters. IEEE Transactions on Knowledge and Data Engineering, 20(11), pp.1441-57.

6. Bohr, N., 1928. The Quantum Postulate and the Recent Development of Atomic Theory1. Nature, 3050(121), pp.580-90.

7. Cunha, D. et al., 2017. Bio-inspired multiobjective clustering optimization: A survey and a proposal. Artificial Intelligence Research, 6(2), pp.10-24.

8. Deb, K., 2001. Multi-objective optimization using evolutionary algorithms. John Wiley \& Sons., 16.

9. Dereli, S. \& Köker, R., 2020. A meta-heuristic proposal for inverse kinematics solution of 7-DOF serial robotic manipulator: quantum behaved particle swarm algorithm. Artificial Intelligence Review, 53(2), pp.949-64.

10. Feynman, R.P., 1982. Simulating physics with computers. International Journal of Theoretical Physics, 21(6-7), pp.467-88.

11. Freitas, A.A., 2004. A critical review of multi-objective optimization in data mining: a position paper. ACM SIGKDD Explorations Newsletter, 6(2), pp.77-86.

12. Fung, B.C., Wang, K. \& Ester, M., 2003. Hierarchical document clustering using frequent itemsets. Proceedings of the 2003 SIAM international conference on data mining. Society for Industrial and Applied Mathematics, pp.59-70.

13. Handl, J. \& Knowles, J., 2007. An evolutionary approach to multiobjective clustering. IEEE transactions on Evolutionary Computation, 11(1), pp.56-76.

14. Heisenberg, P.R., 1929. The Uncertainty Principle. Physical Review, 34(1), pp.163-64.

15. Jain, A.K., 2010. Data clustering: 50 years beyond K-means. Pattern Recognition Letters, 31(8), pp.651-66.

16. Jiacun, W., 2012. Handbook of Finite State Based Models and Applications. CRC press.

17. Jun, S., Wenbo, X. \& Bin, Y., 2006. Quantum-Behaved Particle Swarm Optimization Clustering Algorithm. International Conference on Advanced Data Mining and Applications, pp.340-47.

18. Kennedy, J. \& Eberhart, R., 1995. Particle swarm optimization. Proceedings of ICNN'95-International Conference on Neural Networks IEEE, 4, pp.1942-48. 
19. Lichman, M., 2013. UCI machine learning repository.

20. Matake, N., Hiroyasu, T., Miki, M. \& Senda, T., 2007. Multiobjective clustering with automatic k-determination for large-scale data. Proceedings of the 9th annual conference on Genetic and evolutionary computation, pp.861-68.

21. Mirjalili, S., 2015. Moth-flame optimization algorithm: A novel nature-inspired heuristic paradigm. Knowledge-based systems, 89, pp.228-49.

22. Mukhopadhyay, A., Maulik, U. \& Bandyopadhyay, S., 2015. A Survey of Multiobjective Evolutionary Clustering. ACM Computing Surveys, 47(4), pp.146.

23. Pal, S.K. \& Mitra, S., 1994. Fuzzy versions of Kohonen's net and MLP-based classification: performance evaluation for certain nonconvex decision regions. Information Sciences, 76(3-4), pp.297-337.

24. Saha, S. \& Bandyopadhyay, S., 2012. Some connectivity based cluster validity indices. Applied Soft Computing, 12(5), pp.1555-65.

25. Saha, S. \& Bandyopadhyay, S., 2013. A generalized automatic clustering algorithm in a multiobjective framework. Applied Soft Computing, 13(1), pp.89108.

26. Sun, J., Feng, B. \& Xu, W., 2004. Particle swarm optimization with particles having quantum behavior. Proceedings of the 2004 congress on evolutionary computation (IEEE Cat. No. 04TH8753), 1, pp.325-31.

27. Sun, J., Xu, W. \& Feng, B., 2004. A global search strategy of quantum-behaved particle swarm optimization. IEEE Conference on Cybernetics and Intelligent Systems, 1, pp.111-16.

28. Sun, J., Xu, W. \& Feng, B., 2005. Adaptive parameter control for quantumbehaved particle swarm optimization on individual level. 2005 IEEE international conference on systems, man and cybernetics, 4, pp.3049-54.

29. Wessels, L., 1979. Schrödinger's route to wave mechanics. Studies in History and Philosophy of Science Part A, 10(4), pp.311-40.

30. Yang, S. \& Wang, M., 2004. A quantum particle swarm optimization. In. Proceedings of the 2004 Congress on Evolutionary Computation (IEEE Cat. No. 04TH8753), 1, pp.320-24.

31. Yu, C., Heidari, A.A. \& Chen, H., 2020. A Quantum-behaved Simulated Annealing Enhanced Moth-flame Optimization Method. Applied Mathematical Modelling. 